\title{
Fg Captcha Advanced
}

\author{
Rohith S Aggithaya ${ }^{1}$, Samraddha ${ }^{2}$, Rakshith $\mathbf{M}^{3}$, Karthik Kumar M B \\ Student, Pursuing B.E., Information Science \& Engineering, The National Institute of Engineering, Mysore, India ${ }^{1,2,3,4}$
}

\begin{abstract}
A CAPTCHA (an acronym for "Completely Automated Public Turing test to tell Computers and Humans Apart") is a type of challenge-response test used in computing to determine whether or not the user is human. Most existing CAPTCHAs are text-based. The user is presented with visually distorted text and asked to type it in correctly to prove he or she is a human and not a computer. Due to recent developments in technology, users are rapidly adopting smartphones, tablets, and other non-traditional smart computing devices in lieu of desktop and laptop computers. Many mobile devices lack a physical keyboard, which makes text-based input cumbersome and error-pron. Further, most text based CAPTCHAs are (English) language-dependent and not suitable for multilingual worldwide usage. Some researchers have proposed alternatives to character recognition, in the form of image recognition CAPTCHAs which require users to identify simple objects in the images presented. The unique touch screen technology of mobile devices can be leveraged to create an additional layer of security that is both effective and userfriendly. Fg CAPTCHA proposes a novel image-based CAPTCHA that combines the touch-based input methods favoured by mobile devices with genetically optimized face detection tests to provide a solution that is simple for humans to solve, ready for worldwide use, and provides a high level of security by being resilient to automated computer attacks. It provides a set of embedded images, some of which are photographs of real human faces and others which are cartoons, sketches, or photos of animals representing face-like images to make correctly detecting human faces challenging for computers, complex background pattern designed to confuse the automatic face detection software, thereby increasing the false positive detection rate and a set of visual distortion types (e.g., blurring, contrast adjustment) and the amount of distortion to apply. The proposed genetically optimized fg CAPTCHA works efficiently on both touch screens used by tablets and smartphones and on traditional computers, achieving a high $88 \%$ human accuracy rate during evaluation. It does so without compromising performance, offering an effective $0 \%$ automated attack rate.
\end{abstract}

Keywords: fgcaptcha, existing captcha, newly added features in fgcaptcha , advantages of fg captcha.

\section{INTRODUCTION}

Fg CAPTCHA combines images of human faces, cartoons, Types of CAPTCHAs

and sketches on a multi-colored background. To prevent 1) Web Service

software attacks on the CAPTCHA, two visual distortions 2) Locally-Run

are applied to each CAPTCHA using parameters 3) CSS

optimized by a genetic algorithm. This process ensures the 4) Video CAPTCHA

best possible balance between ease-of-use for humans and 5) Math CAPTCHA

resistance to machine attacks. To solve fg CAPTCHA, users must tap or click on all the human face photographs.In testing, humans were able to successfully solve $87.9 \%$ of fgCAPTCHA images with some distortions have success rates of up to $97 \%$.

As part of the generation process, CAPTCHAs solved by face detection are automatically removed yielding a machine attack rate of approximately 0\%.Graphical passwords were first proposed by Blonder. In his scheme, a password uses an image in which many small regions have been delineated. The user has to choose some of these regions as a password and in order to log in later, the user must click in each of the chosen regions (with a mouse or a stylus). The user must remember the chosen click regions and keep them secret. Several implementations of this idea were given. The information is taken from the paper written by BRIAN M. POWELL, (Member, IEEE), GAURAV GOSWAMI, (Student Member, IEEE), MAYANK VATSA, (Member, IEEE), RICHA SINGH, (Member, IEEE) and AFZEL NOORE, (Senior Member, IEEE)

\section{NEED FOR CAPTCHA}

1) Preventing Comment Spam in Blogs

2) Protecting Website Registration

3) Protecting Email Addresses From Scrapers.

4) Online Polls.

5) Preventing Dictionary Attacks.

6) Search Engine Bots.

7) Worms and Spam

\section{DISADVANTAGE OF TEXT-BASED} CAPTCHA

1) Sometimes very difficult to read

2) Are not compatible with users with disabilities

3) Time-consuming to decipher

4) Technical difficulties with certain internet browsers

5) May greatly enhance Artificial Intelligence.

6) Most text-based CAPTCHAs are (English) languagedependent and not suitable for multilingual worldwide usage. 


\section{EXISTING FGCAPTCHA}

FgCAPTCHA Presents users with a composite image containing several visually distorted human faces along with other objects and non-real faces embedded in a complex background pattern. To prove that a user is human, users must solve the captcha by correctly selecting only the real human faces without choosing any other objects or non-real face images. If this is successfully done, the user is considered to be human and granted access to the secured resource

Features of fgcaptcha:

1) A set of embedded images, some of which are photographs of real human faces and others which are cartoons, sketches, or photos of animals representing face-like images to make correctly detecting human faces challenging for computers.

2) A complex background pattern designed to confuse the automatic face detection software, thereby increasing the false positive detection rate.

3) A set of visual distortion types (e.g., blurring, contrast adjustment) and the amount of distortion to apply, referred to as its intensity.

Steps involved in the generation of fgCAPTCHA image

1. Background Generation

2. Image Selection

3. Distortion Selection

\section{NEWLY ADDED FEATURES TO THE EXISTING FGCAPTCHA}

1) Two levels authentication of fgCAPTCHA. To have two levels of fgcaptcha, after identifying the images in the first sheet go to the next sheet .

2) Identifying the gender of human images displayed on the screen. To identify the gender in the given set of images and write ' $\mathrm{M}$ ' or ' $\mathrm{F}$ 'in the column given below the image.

3) Selecting all the human faces displayed on the screen. Select all the human faces and only human face.

\section{ADVANTAGES OF THE NEWLY ADDED FEATURES IN FGCAPTCHA}

1) The rate at which hacking a system by a software is very low when compared to any other captcha

2) An illiterate can also use this.

\section{CONCLUSION}

The fg CAPTCHA works efficiently on both touch screens used by tablets and smart phones and on traditional computers, achieving a high $88 \%$ human accuracy rate during evaluation. It does so without compromising performance, offering an effective $0 \%$ automated attack rate.

This combination of low attack rates, high human accuracy rates, and convenient mobile device usage provides major improvements over existing desktop centric security CAPTCHAs in widespread use today. The accessing rate of the data by the software is very less compared to any other captcha

\section{ACKNOWLEDGEMENT}

The satisfaction and euphoria that accompanies the successful completion of any task would be incomplete without the mention of the people who made it possible, whose constant guidance and encouragement crowned our effort with success. We would like to express our heartfelt gratitude to those who have given us their unconditional support and helped us throughout this endeavour. First and foremost we would like to thank Dr. G. L. Shekar, Principal, NIE, Mysore, for his moral support towards completing our project work. We deeply express our sincere gratitude to our guide Mrs. Prajakta M, Asst. Professor, Department of Information Science and Engineering, N.I.E, Mysore for her valuable guidance and Dr. K Raghuveer, Professor and Head, Department of Information Science \& Engineering, NIE, Mysore, for guidance, constant encouragement, support and suggestions for improvement. We would like to express our deepest gratitude to our family members, for their support and love. Finally, we would like to thank all our friends, who all made invaluable contributions to our work.

\section{REFERENCES}

[1]. fgCAPTCHA: Genetically Optimized Face Image CAPTCHA BRIAN M. POWELL1, (Member, IEEE), GAURAV GOSWAMI2, (Student Member, IEEE), MAYANK VATSA2, (Member, IEEE), RICHA SINGH2, (Member, IEEE), AND AFZEL NOORE1, (Senior Member, IEEE)

[2]. R. A. Botha, S. M. Furnell, and N. L. Clarke, “'From desktop to mobile: Examining the security experience," Computer. Security, vol. 28, nos. 34, pp. 130137, 2009.

[3]. J.-C. Birget, D. Hong, and N. Memon, "Graphical passwords based on robust discretization," IEEE Trans. Inf. Forensics Security, vol. 1 , no. 3 , pp. 395399 , Sep. 2006 